\title{
Vibrational-Resonance Enhancement of Positron Annihilation in Molecules
}

\author{
S. J. Gilbert, L. D. Barnes, J. P. Sullivan, and C. M. Surko \\ Department of Physics, University of California, San Diego, California 92093-0319
}

(Received 8 August 2001; published 11 January 2002)

\begin{abstract}
The rate of annihilation of low-energy positrons in many molecular gases is orders of magnitude larger than can be explained on the basis of simple collisions. Developments in positron beam technology have enabled the first energy-resolved measurements of this annihilation process. The results of these experiments provide direct evidence that the large observed values of annihilation rate are due to the excitation of long-lived vibrational resonances of the positron-molecule complex. These results are generally consistent with a recent theoretical model of resonant annihilation.
\end{abstract}

DOI: $10.1103 /$ PhysRevLett.88.043201

The interaction of positrons with matter is important in many areas of science and technology. Examples include understanding gamma-ray spectra of astrophysical origin [1], positron binding to atoms [2], positron-induced ionization and fragmentation of molecules [3], and the characterization of thin films, materials, and material surfaces [4]. One outstanding problem in this area, first pointed out in a seminal paper by Paul and Saint-Pierre [5], is that positron annihilation rates in molecular gases, at energies below the threshold for positronium formation, can be orders of magnitude larger than those expected on the basis of simple collisions. This phenomenon, which has yet to be understood, is important both in establishing the basis of a chemistry of matter and antimatter and in determining many potential uses of positrons.

In this Letter, we present the first study of positron annihilation as a function of positron energy. The work exploits the recently developed ability to create a cold positron beam tunable over a wide energy range [6]. While this beam has been used to make new positron scattering measurements [7], this Letter focuses on the study of positron annihilation. The results provide direct evidence that vibrational resonances of the positron-molecule complex are responsible for the greatly enhanced molecular annihilation rates. The results also provide evidence that positrons bind to molecules.

Positron annihilation rates for atoms and molecules are conventionally expressed in terms of a dimensionless parameter, $Z_{\text {eff }}$, which is the measured annihilation rate, $\Gamma$, divided by that calculated for an uncorrelated electron gas at a density equal to the molecular number density, $n_{m}$. Thus, $Z_{\text {eff }}=\Gamma /\left(r_{0}^{2} c n_{m}\right)$, where $r_{0}$ is the classical radius of the electron and $c$ is the speed of light. If there were no correlation between the positron and the molecular electrons, $Z_{\mathrm{eff}}$ would be equal to the number of electrons, $Z$, in the molecule. Yet in butane, for example, $Z_{\text {eff }}$ is approximately $10^{4}$, which is more than 2 orders of magnitude larger than $Z$ [5,8-11]. Values as large as $Z_{\text {eff }} \sim 10^{6}-10^{7}$ are observed for alkane molecules only 2 to 3 times larger than butane [8].

Extensive experimental studies of this phenomenon can be found in Refs. [8-13]. Most of these studies were
PACS numbers: $34.85 .+\mathrm{x}, 34.50 .-\mathrm{s}, 71.60 .+\mathrm{z}, 78.70 . \mathrm{Bj}$

done with a Maxwellian distribution of positrons at $300 \mathrm{~K}$ (i.e., equivalent to $25 \mathrm{meV}$ ). Although many chemical trends have been identified, and it has been determined that the annihilation occurs with roughly equal probability on any valence electron [12], the mechanism responsible for these greatly enhanced annihilation rates has remained an unresolved problem for over three decades.

Since the work of Paul and Saint-Pierre, there have been several proposals to explain large values of $Z_{\mathrm{eff}}$ in terms of some kind of electronic or vibrational resonance or positron-molecule bound state [10,13-19]. The presence of either a resonance or bound state is expected to result in a long-lived positron-molecule complex, thereby increasing the overlap of the positron and electron wave functions and greatly enhancing the probability of annihilation.

A comprehensive model of this phenomenon, developed recently by Gribakin $[13,16]$, considers two mechanisms. Values of $Z_{\text {eff }} \leq 10^{3}$, such as those observed for small molecules, are explained in terms of virtual or weakly bound states that involve the correlated motion of the positron and the molecular electrons. However, annihilation rates, $Z_{\text {eff }} \geq 10^{3}$, observed in larger molecules are attributed to positron capture in vibrational Feshbach resonances (i.e., resonances involving the motion of the atomic nuclei). In the framework of the model, the latter process (and hence $Z_{\text {eff }} \geq 10^{3}$ ) is possible only for molecules that have bound states with the positron. An incident lowenergy positron can then excite a vibrational mode of the positron-molecule complex and become temporarily bound to the molecule, greatly increasing the probability of annihilation. Since the number of vibrational resonances increases very rapidly with molecular size, the ability of the positron to couple to this large number of resonances provides a natural explanation for the rapid increase of $Z_{\text {eff }}$ that is observed as the size of the molecule is increased.

There have also been proposals $[15,17,19]$ and further discussion $[8,20,21]$ of models that do not involve vibrational resonances. These models were motivated, in part, by the observation that large values of $Z_{\text {eff }}$ correlate with the energies of the electronic states of the molecules [11]. They involve concepts such as highly correlated electronpositron states, the excitation of virtual positronium, and 
generic behavior at inelastic thresholds, all of which are expected, at least to some degree, to increase the overlap of the positron and electron wave functions and increase $Z_{\text {eff }}$.

The net result is that, until now, confirmation of the physical mechanism responsible for large $Z_{\text {eff }}$ in molecules has been lacking. This paper describes the results of highresolution measurements of $Z_{\text {eff }}$ as a function of positron energy spanning the range of energies of the molecular vibrations. These measurements provide direct evidence that the excitation of vibrational resonances of the positron-molecule complex is responsible for the greatly enhanced values of $Z_{\text {eff }}$.

The experiment is shown schematically in Fig. 1. Positrons are accumulated and cooled to $300 \mathrm{~K}$ in a specially designed three-stage, Penning-Malmberg, buffer-gas trap, operating at a magnetic field of $0.15 \mathrm{~T}[10,22]$. A mixture of $\mathrm{N}_{2}$ and $\mathrm{CF}_{4}$ is used for rapid cooling of the positrons to $300 \mathrm{~K}$. The cold positrons are forced over a potential barrier to form a beam tunable in energy from $50 \mathrm{meV}$ upward, with a parallel energy resolution of $25 \mathrm{meV}$, FWHM [6]. To minimize the effects of space charge on the energy resolution of the beam, the experiment is conducted with $3 \mu$ s pulses of $\simeq 5 \times 10^{4}$ positrons at a $4 \mathrm{~Hz}$ rate. This beam is magnetically guided through an annihilation cell containing the test gas. The adjustable voltage, $V_{c}$, on the cell and the exit gate potential, $V$, on the trap determine the positron beam
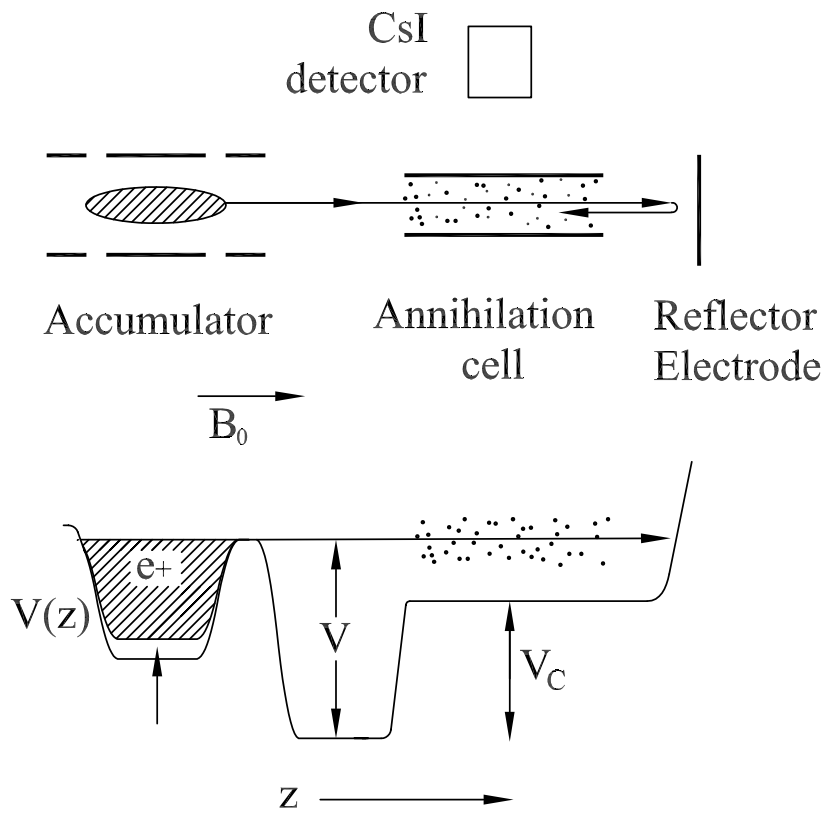

FIG. 1. Schematic diagram of the experiment (above), and corresponding electrical potential $V(z)$ as a function of position along the magnetic field (below). A cold positron beam is passed through a gas cell. The energy of the beam, $\varepsilon$, can be tuned from $50 \mathrm{meV}$ to several electronvolts. To avoid background, the positrons are kept in flight while annihilation events are recorded. energy, $\varepsilon=e\left(V-V_{c}\right)$. There is a continuous flow of test gas through the cell which is typically maintained at a pressure $\sim 0.02$ mtorr, measured to an uncertainty $\sim 15 \%$. In the absence of the test gas, the cell can be used as a retarding potential energy analyzer to measure the parallel energy and energy spread of the beam. The range of $\varepsilon$ studied is from $50 \mathrm{meV}$ to several electronvolts.

A specially designed CsI gamma-ray detector with associated light pipe and photodiode is used to detect annihilation events from a $10 \mathrm{~cm}$ region along the positron beam in the gas cell. The low level of the annihilation signal (e.g., $\sim 0.05$ counts per positron pulse) requires virtually perfect shielding from stray gamma rays. As illustrated in Fig. 1, gamma rays that would arise if the positrons were to strike a collector plate or other surface are avoided by keeping the positrons in flight between the trap and the opposite end of the gas cell while the annihilation signal is recorded (typically $\sim 10$ round trips through the cell in $20 \mu \mathrm{s}$ ). Background gamma-ray sources, including cosmic radiation and stray annihilations from the ${ }^{22} \mathrm{Na}$ positron source, are reduced by a $5 \mathrm{~cm}$ thick lead shielding surrounding the annihilation cell and the CsI detector. The background is further reduced by collecting data only during the $20 \mu \mathrm{s}$ interval that the positrons are in flight. The detector is operated in a pulse counting mode to detect single annihilation gamma rays at $511 \mathrm{keV}$. The number of annihilations per positron transit through the cell; the measured gas pressure in the cell and $\varepsilon$ are used to calculate $Z_{\text {eff }}$. The annihilation signal was verified to be linear in test gas pressure and in the number of transits through the cell. The noise in the data is statistical and proportional to the square root of the number of detected gamma rays. Uncertainties in the absolute values of the $Z_{\text {eff }}$ measurements are estimated to be $\pm 25 \%$, taking into account uncertainties in the gas pressure, number of positrons per pulse, and the detector efficiency.

Shown in Fig. 2(a) are data for butane $\left(\mathrm{C}_{4} \mathrm{H}_{10}\right)$. There is a large annihilation signal in the region of the molecular vibrational modes. The most prominent feature is a large peak with $Z_{\text {eff }}=23000$, occurring at an energy of $0.33 \mathrm{eV}$, which is $\Delta \varepsilon \simeq 30 \mathrm{meV}$ below the $\mathrm{C}-\mathrm{H}$ vibrational stretch modes in butane at $\simeq 0.36 \mathrm{eV}$. The width of the peak is $\simeq 40 \mathrm{meV}$ FWHM, which is about a factor of 2 larger than the resolution of the positron beam. To confirm that the observed peak is due to the $\mathrm{C}-\mathrm{H}$ stretch mode, similar data were taken for deuterated butane (d-butane, $\mathrm{C}_{4} \mathrm{D}_{10}$ ), which exhibits a peak, with $Z_{\text {eff }}=$ 28500 at an energy of $0.23 \mathrm{eV}$. While d-butane has the same electronic structure as butane, the C-D stretch mode is lower in energy by a factor $\xi^{1 / 2}$, where $\xi=\mu_{\mathrm{C}-\mathrm{D}} / \mu_{\mathrm{C}-\mathrm{H}}=1.86$ is the ratio of the reduced masses associated with these vibrational modes. As shown by the dashed curve in Fig. 2(a), not only the positions and shapes of the $\mathrm{C}-\mathrm{H}$ and $\mathrm{C}-\mathrm{D}$ peaks, but the entire spectra for butane and d-butane agree quite well when the d-butane spectrum is scaled in energy by $\xi^{1 / 2}$. 


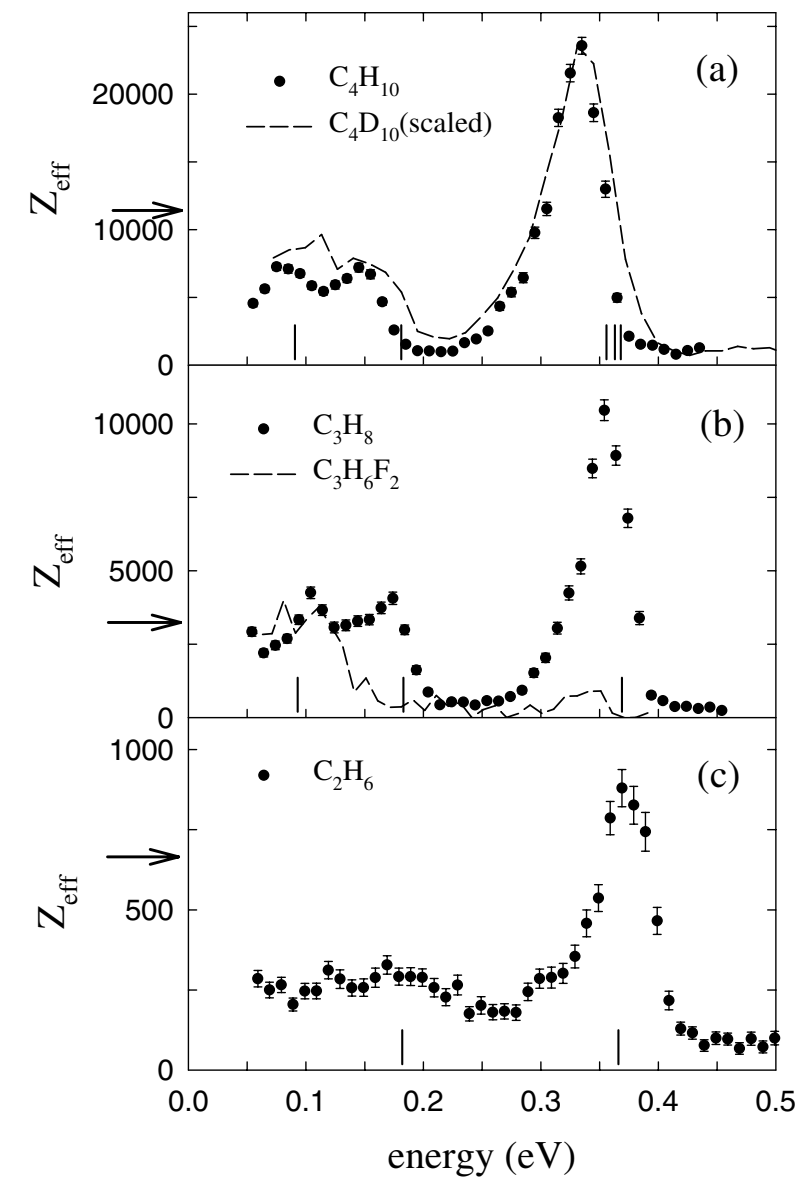

FIG. 2. Positron annihilation rate, $Z_{\text {eff }}$, for (a) butane, (b) propane, and (c) ethane, as a function of positron energy in the range, $50 \leq \varepsilon \leq 450 \mathrm{meV}$. Vertical bars along the abscissas indicate the strongest infrared-active vibrational modes. Arrows on the ordinate indicate $Z_{\text {eff }}$ for a Maxwellian distribution of positrons at $300 \mathrm{~K}$. $Z_{\text {eff }}$ for d-butane is shown by the dashed curve in (a), with the amplitude normalized, and the energy scaled by the appropriate reduced-mass factor (see text). The annihilation rate for 2,2-difluoropropane $\left(\mathrm{C}_{3} \mathrm{H}_{6} \mathrm{~F}_{2}\right)$ is indicated by the dashed curve in (b).

Data for two other alkane molecules, propane $\left(\mathrm{C}_{3} \mathrm{H}_{8}\right)$ and ethane $\left(\mathrm{C}_{2} \mathrm{H}_{6}\right)$, are shown in Fig. 2. They exhibit spectra similar to butane, with $\mathrm{C}-\mathrm{H}$ stretch peak values $Z_{\text {eff }} \simeq 10500$ and 900 , respectively, and large values of $Z_{\text {eff }}$ throughout much of the range of the molecular vibrations. The downward shift in the location of the peak relative to the $\mathrm{C}$ - $\mathrm{H}$ mode is $\Delta \varepsilon \simeq 20 \mathrm{meV}$ for propane and zero for ethane, to within the accuracy of the measurements $( \pm 15 \mathrm{meV})$. In Fig. 3, annihilation rates, $Z_{\mathrm{eff}}$, are shown for the two carbon molecules, (a) ethylene $\left(\mathrm{C}_{2} \mathrm{H}_{4}\right)$ and (b) acetylene $\left(\mathrm{C}_{2} \mathrm{H}_{2}\right)$. The maximum $Z_{\text {eff }}$ values are 1100 and 1400 , respectively, and the $\mathrm{C}-\mathrm{H}$ stretch mode no longer dominates the spectra as it did in Fig. 2.

Fluorination of hydrocarbons is known to produce large changes in $Z_{\text {eff }}[8,13]$. Data for 2,2-difluoropropane $\left(\mathrm{C}_{3} \mathrm{H}_{6} \mathrm{~F}_{2}\right)$ are indicated in Fig. $2(\mathrm{~b})$, showing that the addition of two fluorine atoms to the molecule causes the resonant $\mathrm{C}-\mathrm{H}$ peak in propane to disappear.

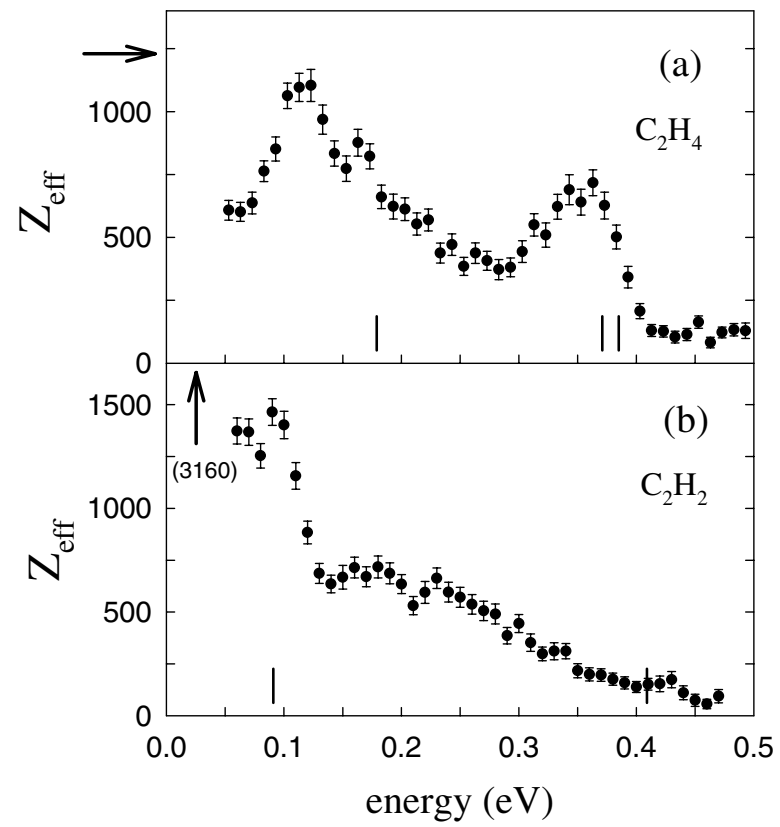

FIG. 3. Positron annihilation rates, $Z_{\text {eff }}$, for the two carbon molecules, (a) ethylene and (b) acetylene. Vertical bars along the abscissas and the arrows on the ordinate are as in Fig. 2.

For all molecules studied, values of $Z_{\text {eff }}$ at energies above those of the vibrational modes (e.g., $0.4<\varepsilon \leq$ several electron volts) were found to be much smaller (e.g., at a level $Z_{\text {eff }} \sim 100$ ) than the values measured at lower energies.

The large increases in $Z_{\text {eff }}$ observed as the beam energy is tuned through the energy range of the molecular vibrations in butane, propane, and ethane provide direct evidence that the annihilation occurs via vibrational resonances of the positron-molecule complexes. In all of the molecules studied, the value of $Z_{\text {eff }}$ measured for a Maxwellian distribution of positrons at $300 \mathrm{~K}$ is of the order of the average value of $Z_{\text {eff }}$ measured throughout the energy range of the vibrational modes $(0.05-0.4 \mathrm{eV})$ and is much greater than $Z$. These room-temperature values of $Z_{\text {eff }}$ are indicated by arrows on the ordinates in Figs. 2 and 3 . The ratios of the maximum values of $Z_{\text {eff }}$ at the $\mathrm{C}-\mathrm{H}$ stretch mode peak to the values at $300 \mathrm{~K}$ are 2 for butane, propane, and ethane, even though the values of $Z_{\text {eff }}$ vary by more than an order of magnitude. These comparisons provide evidence that the same (or a very similar) resonant annihilation mechanism determines $Z_{\text {eff }}$, both in the energy range of the molecular vibrations and at $300 \mathrm{~K}$.

The results presented here support previous conjectures as to the importance of vibrational resonances in producing large $Z_{\text {eff }}[10,14]$, and they confirm specific predictions of the vibrational resonance model [16,23]. This theory indicates that values of $Z_{\text {eff }} \leq 10^{3}$ at $300 \mathrm{~K}$ can be explained without excitation of vibrational resonances, while larger values of $Z_{\text {eff }}$ cannot [16,23]. This is qualitatively consistent with the data in that large vibrational enhancements are seen for molecules with $Z_{\text {eff }}(300 \mathrm{~K})>1000$. 
Furthermore, if a positron-molecule complex has a bound state, a vibrational resonance in the complex is expected to occur at an energy lower by the amount of the binding. Thus the downward shift in the resonance peak in butane of $\Delta \varepsilon \simeq 30 \mathrm{meV}$ provides the most direct evidence to date that positrons can bind to molecules. The existence of such a bound state is a key assumption of the model. Finally, the positron-molecule potential that produces this binding in hydrocarbons is predicted to become less attractive with the substitution of fluorine atoms [13,16,23]. Measurements of $Z_{\text {eff }}(\varepsilon)$ for difluoropropane $\mathrm{C}_{3} \mathrm{H}_{6} \mathrm{~F}_{2}$ [Fig. 2(b), dashed line] show a much smaller amplitude at the $\mathrm{C}-\mathrm{H}$ peak than is observed in propane, and this is also consistent with the model [16].

The ability, demonstrated here, to study in detail the behavior of $Z_{\text {eff }}$ at vibrational resonances will allow new and precise tests of theories of annihilation in molecules. For the butane data shown in Fig. 2, estimates using the resonant annihilation formulas indicate that the $\mathrm{C}-\mathrm{H}$ peak is larger by a factor of $\sim 7$ than can be explained by a single resonance [23]. A peak of this magnitude could, however, be due to several unresolved resonances, which might be populated either directly or by nonlinear coupling $[16,23]$. Another point is obtaining a quantitative understanding of $Z_{\text {eff }}(300 \mathrm{~K})$. As can be seen from Figs. 2 and 3 and pointed out above, values of $Z_{\text {eff }}$ at energies in the range from 50 to $200 \mathrm{meV}$ are comparable to $Z_{\mathrm{eff}}(300 \mathrm{~K})$. While $Z_{\text {eff }}$ was measured only in the present experiment down to $50 \mathrm{meV}$, measurements of $Z_{\mathrm{eff}}$ in butane as a function of positron temperature, $T_{p}$ [13], show that $Z_{\mathrm{eff}}\left(T_{p}\right)$ increases by about $60 \%$ as $T_{p}$ is decreased from $600 \mathrm{~K}$ (i.e., $50 \mathrm{meV}$ ) to $300 \mathrm{~K}$, varying as $T_{p}^{-1 / 2}$. The origin of this scaling is unclear. Also unclear is the previously observed empirical scaling [11] of $\ln \left[Z_{\text {eff }}(300 \mathrm{~K})\right]$ with $\left(E_{i}-E_{P S}\right)^{-1}$, where $E_{i}$ is the ionization energy of the molecule and $E_{P s}=6.8 \mathrm{eV}$ is the positronium formation energy. A final point for further consideration is the lack of expected changes of $Z_{\text {eff }}(300 \mathrm{~K})$ on substitution of deuterium for hydrogen atoms in large hydrocarbons [13].

Knowledge of the binding energy of positrons to molecules (e.g., butane) could be used to make a more accurate prediction of $Z_{\text {eff }}(\varepsilon)$ including comparison with the $300 \mathrm{~K}$ data. While the study described here focused on hydrocarbon molecules of modest size, the resonance annihilation phenomenon is expected to be quite general. Thus similar studies of other large molecules are likely to add further insight into the nature of the positron molecule complexes discussed here and the mechanism responsible for large annihilation rates.

We have presented data that establish for the first time the importance of vibrational resonances in producing the large positron annihilation rates observed in wide classes of molecules. As antimatter becomes more readily available in the laboratory, understanding this and similar phenomena can be expected to become more important in re- alizing a range of scientific and technological capabilities, from studies of fundamental symmetries of nature to the selective ionization of biomolecules and the characterization of materials.

We are pleased to acknowledge helpful conversations with Gleb Gribakin and Steve Buckman and the technical assistance of Gene Jerzewski. This work is supported by National Science Foundation Grant No. PHY 98-76894 and the Office of Naval Research, Grant No. N00014-97-1-0366.

[1] R. Ramaty and R.E. Lingenfelter, in High Energy Astrophysics, edited by J. Matthews (World Scientific, New York, 1994), p. 32.

[2] G. G. Ryzhikh and J. Mitroy, Phys. Rev. Lett. 79, 4124 (1997).

[3] L. D. Hulett, Jr., D. L. Donohue, J. Xu, T. A. Lewis, S. A. McLuckey, and G. L. Glish, Chem. Phys. Lett. 216, 236 (1993).

[4] P. J. Schultz and K. G. Lynn, Rev. Mod. Phys. 60, 701 (1988).

[5] D. A. L. Paul and L. Saint-Pierre, Phys. Rev. Lett. 11, 493 (1963).

[6] S. J. Gilbert, C. Kurz, R. G. Greaves, and C. M. Surko, Appl. Phys. Lett. 70, 1944 (1997).

[7] J. P. Sullivan, Phys. Rev. Lett. 87, 073201 (2001); 86, 1494 (2001).

[8] K. Iwata, R. G. Greaves, T. J. Murphy, M. D. Tinkle, and C. M. Surko, Phys. Rev. A 51, 473 (1995).

[9] G. R. Heyland, M. Charleton, T. C. Griffith, and G. L. Wright, Can. J. Phys. 60, 503 (1982).

[10] C. M. Surko, A. Passner, M. Leventhal, and F. J. Wysocki, Phys. Rev. Lett. 61, 1831 (1988).

[11] T. J. Murphy and C. M. Surko, Phys. Rev. Lett. 67, 2954 (1991).

[12] K. Iwata, Phys. Rev. A 55, 3586 (1997).

[13] K. Iwata, G. F. Gribakin, R. G. Greaves, C. Kurz, and C. M. Surko, Phys. Rev. A 61, 022719 (2000).

[14] P. M. Smith and D. A.L. Paul, Can. J. Phys. 48, 2984 (1970).

[15] G. Laricchia and C. Wilkin, Phys. Rev. Lett. 79, 2241 (1997).

[16] G. F. Gribakin, Phys. Rev. A 61, 022720 (2000).

[17] E. P. da Silva, J. S. E. Germane, and M. A. P. Lima, Phys. Rev. Lett. 77, 1028 (1996).

[18] V. I. Goldanskii and Y. S. Sayasov, Phys. Lett. 13, 300 (1964).

[19] G. Laricchia and C. Wilkin, Nucl. Instrum. Methods Phys. Res., Sect. B 143, 135 (1998).

[20] J. Mitroy, M. W. J. Bromley, and G. Ryzhikh, J. Phys. B 32, 2203 (1999).

[21] G. G. Ryzhikh and J. Mitroy, J. Phys. B 33, 2229 (2000).

[22] R. G. Greaves and C. M. Surko, Phys. Plasmas 4, 1528 (1997).

[23] G. F. Gribakin, in New Directions in Antimatter Chemistry and Physics, edited by C. M. Surko and F. A. Gianturco (Kluwer Academic Publishers, The Netherlands, 2001), p. 366; (private communication). 